1964; the second is a thoroughly updated and somewhat expanded version. The book has the characteristics both of a textbook, with its detailed didactic presentation of theory, and of a review with its numerous references to the literature. This dual objective is the main difficulty of the book, for with so large a subject the material has become so bulky that its practical usefulness is jeopardized. The author is aware of the size of the field and of the existence of many smaller works dealing with specialized areas, but he is also aware of the value of a coherent presentation of a subject by a single author. He consciously offers his book, therefore, as giving, 'as before, a one-man view of solid-state chemistry'.

The new edition has essentially the same structure as the first but the three parts of the original book have become three separately bound volumes. The first volume (which appeared ahead of the other two, about six months ago) deals with preparative procedures and the theory of crystal growth. A good deal of basic thermodynamics is included. The second volume, the largest of the three, deals with imperfections (including impurities) in crystalline materials, and their physico-chemical consequences. The first three chapters of this volume are particularly useful and readable - as a survey of the nature of crystal imperfections in general. Subsequent chapters develop the detailed physical chemistry and mathematics of equilibria involving imperfections of all possible kinds, and then examine the application of such analysis to increasingly complex substances, from elements, through a substantial range of binary compounds to the more important tertiary compounds. The third volume is a study of all situations which are specifically not in equilibrium, i.e. relaxation effects. chemical changes or reactions in or on solids. This includes sections on the diffusion of crystal imperfections, precipitation of impurities, the mechanisms of sintering and tarnishing, the electrochemistry of imperfect solids, the photographic process and charge-transfer catalysis. This third volume is perhaps the most useful to the active research worker. Through the three volumes, the numbering of the chapters runs sequentially but the page numbering does not, which seems inconsistent; also, the three indexes are quite separate. Cross-referencing within the three volumes is frequent but between them rather rare.
The thoroughness with which the literature citations have been updated is impressive, and reflects also the expansion of the subject during the past decade. A useful table in Vol. 1 lists all substances which have been grown as single crystals, with the methods used and the literature references. In the first edition, this contained about 200 entries and 230 references; it now extends over 38 pages, has 700 entries and 1100 references. Only molecular solids (e.g. organic crystals) are conspicuous by their absence from these compilations. Mammoth tables are a feature of this book: a table in Vol. 2, listing binary compounds for which any work has been carried out on the influences of impurities on crystal properties, runs to 86 pages and has 3400 references. There are many such enormous tabulations. They do not make for smooth reading of the book but they certainly contain a wealth of information, allowing the reader instant access to the original literature at whatever point he likes.

A vital contribution that Kröger has made to the subject (in addition to his own research work in this field) is the symbolism he has devised for crystal imperfections. This is presented at the beginning of Vol. 2 and is used continuously throughout the book. There is a useful summary on p. 275, at the end of Vol. 3. Characteristically it is rather elaborate, so as to allow for all possible situations. Perhaps it is because it has appeared cumbersome that it has not been widely adopted, but it is in fact an elegant and practical scheme, worth knowing and using

Misprints are remarkably few, considering the size and complexity of this book (although there is a bad typographical displacement on p. 469 of Vol. 2). There is one serious infelicity in the occasional use of the term free enthalpy' for the Gibbs free energy, $G$. Free energy relationships play an important part in this book, so it is a pity that this invitation to confusion is presented. 'Free enthalpy' is neither defined nor explained as such; indeed, the impression that it is being used interchangeably with the normal term is fortified by duplicated index entries. If 'free enthalpy' was merely a verbal indiscretion, then it is curious that this was not corrected during re-editing, when linguistic slips such as 'specie' were removed. As it stands it is misleading, for $G$ is emphatically not a sort of enthalpy.
Taken as a whole the book is excellent in terms of clarity, coherence and internal consistency. Its width of coverage is masterly, even encyclopaedic; its accurate presentation of detail is remarkable. As a reference text, giving both the broad panoramic view and a meticulous literature survey, it must be unique in the field. Only its cost is unfortunate, for at $\mathrm{f} 70$ one can hardly expect the book to be purchased by ordinary individuals. Any of the three volumes can of course be bought separately but this is not really to be recommended, for the work is intended as a single treatise. However, many libraries must surely acquire this book and many working scientists and technologists will find invaluable its compendious stock of organized information.

J. H. ROBERTSON

School of Chemistry

University of Leeds

England

\title{
Book Received
}

The following book has been received by the Editor. Brief and generally uncritical notices are given of works of marginal crystallographic interest; occasionally a book of fundamental interest is included under this heading because of difficulty in finding a suitable reviewer without great delay.

Handbook of Geochemistry. Executive Editor: K.H.WEDEPOHL. Editorial Board: C.W. Correns, D. M. Shaw, K. K. Turekian, J. JeMANN. Vol. 2, Part 4. Pp. VI + 898, Figs. 113. Berlin, Heidelberg, New York: Springer - Verlag, 1974. Loose-leaf binder DM 298,00 , US $\$ 122.20$. Subscription price for purchases of $\mathrm{Vol}$. 2: Loose-leaf binder DM238,40, US $\$ 97.80$.

This is the fourth part of a work which is produced in loose-leaf form for assembly by the user. The chapters are numbered according to the atomic numbers of the elements and the present instalment either completes or supplies in complete form the following chapters:

Boron 5, Carbon 6, Nitrogen 7, Oxygen 8, Fluorine 9, Magnesium 12, Silicon 14, Chlorine 17, Vanadium 23, Iron 26. Copper 29, Zinc 30, Gallium 31, Selenium 34, Bromine 35, Silver 47, Antimony 51 , Tellurium 52, lodine 53, Barium 56, Tungsten (Wolfram) 74, Gold 79. Thallium 81 and Lead 82. 